\title{
Adsorption and aggregation of monoclonal antibodies at silicone oil-water interfaces
}

\author{
Aadithya Kannan ${ }^{1,2}$, lan C. Shieh², Patrick G. Negulescu ${ }^{3}$, Vineeth Chandran Suja ${ }^{1}$, Gerald G. Fuller ${ }^{1}$ \\ ${ }^{1}$ Stanford University, Stanford, California, USA \\ ${ }^{2}$ Genentech, South San Francisco, California, USA \\ ${ }^{3}$ University of California, Berkeley, California, USA
}

\section{Supplementary Information}

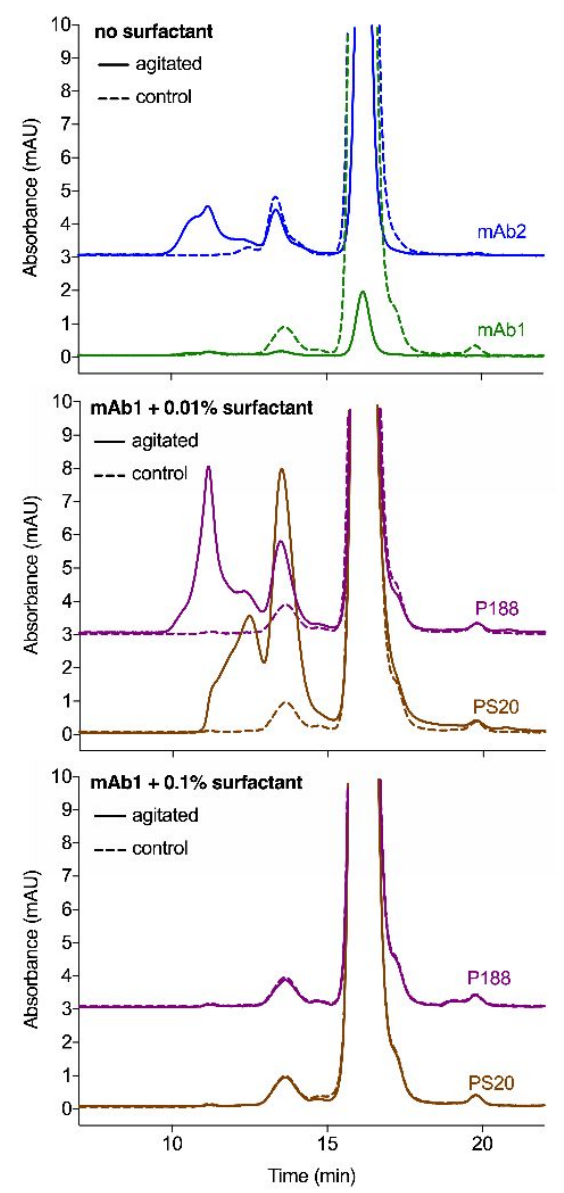

Figure S1. Chromatograms obtained through SE-HPLC. The dashed lines are the unagitated controls and the solid lines are the agitated samples. Top panel - mAb1 and mAb2 solutions, middle panel - mAb1 + 0.01\% P188/PS20, bottom panel - mAb1 + 0.1\% P188/PS20. 

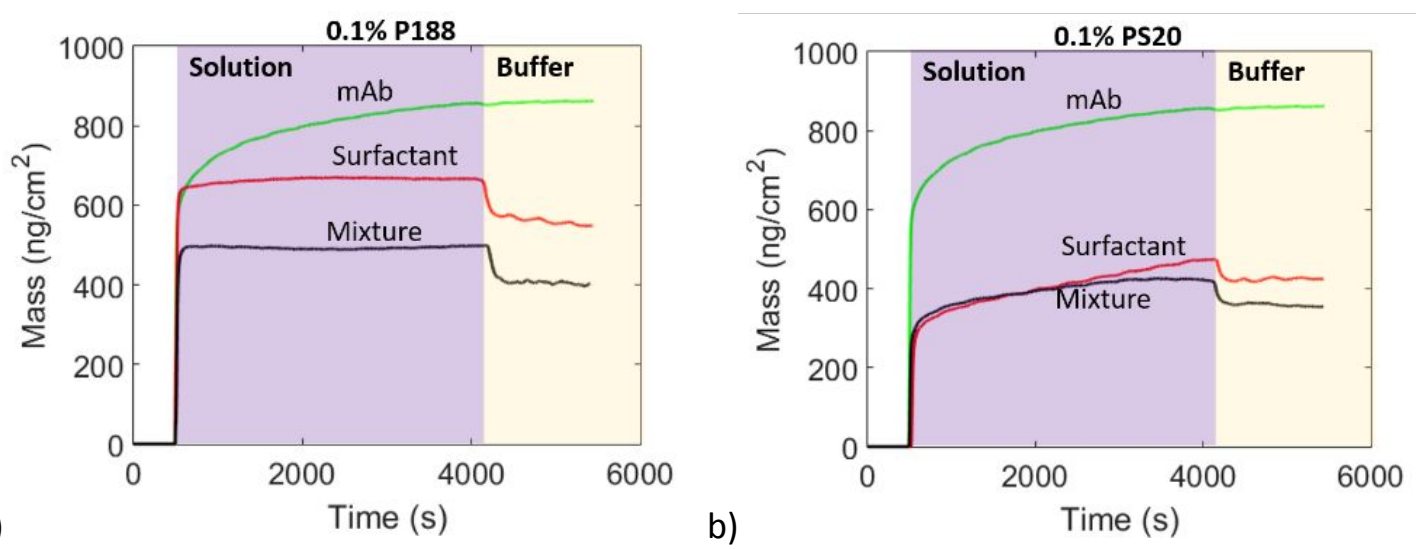

Figure S2. a,b) Mass adsorbed per unit area measured using quartz crystal microbalance for pure mAb1, pure surfactant at $0.1 \%$ and $\mathrm{mAb}+$ surfactant mixture. a) mAb with $0.1 \% \mathrm{P} 188$. The mass of the surfactant adsorbed was greater as compared to P188 at $0.01 \%$ (Fig. 4a). The mixture's adsorption was similar to the surfactant, but slightly lower as the presence of mAbs lowered the surfactant packing density, similar to what was seen at lower concentrations. b) mAb with $0.1 \%$ PS20. The mixture had very similar adsorption as the pure surfactant case.
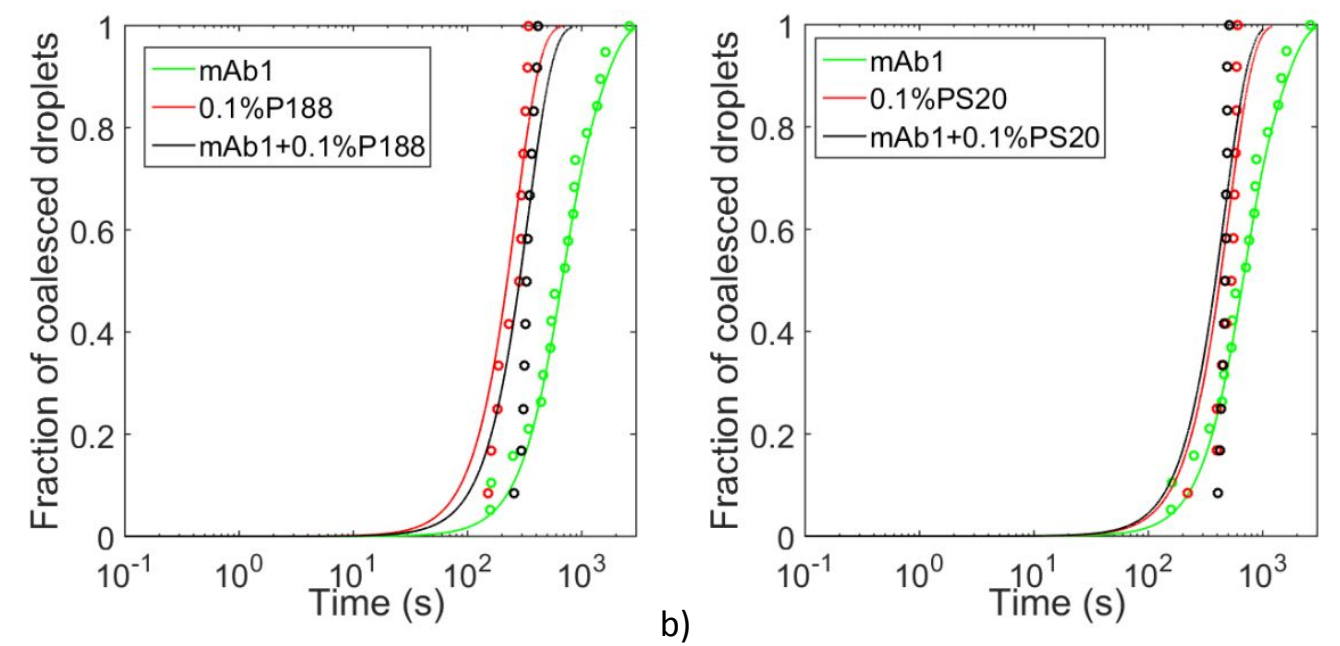

Figure S3. a,b) Cumulative distribution of coalescence times of oil droplets with a flat oil-water interface a) $\mathrm{mAb}$ with $0.1 \% \mathrm{P} 188$. b) mAb with $0.1 \% \mathrm{PS} 20$. In both cases, the coalescence times of the surfactants were longer than those at $0.01 \%$ concentrations (Fig. 4 c,d). And the mixture had coalescence times similar to the pure surfactant case, as the surfactants dominated the interfaces in both cases. It was also observed that the coalescence times were deviating from the Rayleigh distribution as they were more deterministic with a better packing of surfactants at the interface. 


\begin{tabular}{|c|c|c|c|c|c|c|}
\hline \multirow[b]{2}{*}{$\begin{array}{l}\text { Surfactant } \\
\text { Type }\end{array}$} & \multirow[b]{2}{*}{$\begin{array}{c}\text { Surfactant } \\
\text { Level }(\% \mathrm{w} / \mathrm{v})\end{array}$} & \multirow[b]{2}{*}{ Time Point } & \multicolumn{4}{|c|}{ SE-HPLC } \\
\hline & & & (\% HMWS) & $\begin{array}{c}\text { (\% Main } \\
\text { Peak) }\end{array}$ & (\% LMWS) & $\begin{array}{c}\text { Total peak area } \\
\text { (mAU*min) }\end{array}$ \\
\hline mAb1 & 0 & No agitation & 1.8 & 97.8 & 0.4 & 56.6 \\
\hline mAb1 & 0 & $0.5 \mathrm{~h}$ agitation & 20.4 & 79.6 & 0 & 1.4 \\
\hline mAb1/PS20 & 0.01 & No agitation & 1.6 & 98 & 0.4 & 59 \\
\hline mAb1/PS20 & 0.01 & $0.5 \mathrm{~h}$ agitation & 24 & 75.4 & 0.6 & 47.5 \\
\hline mAb1/PS20 & 0.1 & No agitation & 1.6 & 98 & 0.4 & 62.1 \\
\hline mAb1/PS20 & 0.1 & $0.5 \mathrm{~h}$ agitation & 1.6 & 98 & 0.4 & 60 \\
\hline mAb1/P188 & 0.01 & No agitation & 1.6 & 98 & 0.4 & 59.2 \\
\hline mAb1/P188 & 0.01 & $0.5 \mathrm{~h}$ agitation & 15.8 & 83.6 & 0.6 & 45.6 \\
\hline mAb1/P188 & 0.1 & No agitation & 1.6 & 97.8 & 0.6 & 59.3 \\
\hline mAb1/P188 & 0.1 & $0.5 \mathrm{~h}$ agitation & 1.5 & 97.9 & 0.6 & 56.8 \\
\hline mAb2 & 0 & No agitation & 2.6 & 97.3 & 0.1 & 54.5 \\
\hline mAb2 & 0 & $0.5 \mathrm{~h}$ agitation & 25.2 & 74.7 & 0.1 & 12.5 \\
\hline
\end{tabular}

Table S1. Results from SE-HPLC (SEC) used for calculating the \% loss of monomers, change in \% of oligomeric aggregates and the \% change in SEC total peak area. 